\title{
Suppression of Polysulfide Dissolution and Shuttling with Glutamate Electrolyte for Lithium Sulfur Batteries
}

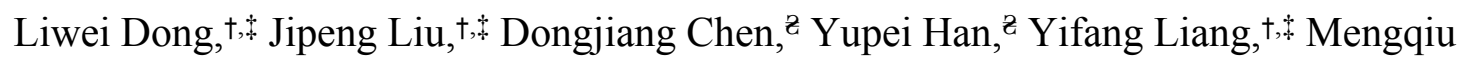
Yang,,$*$ Chunhui Yang, ${ }^{*, \dagger}$ and Weidong $\mathrm{He}^{*, \ddagger, z}$

†School of Chemistry and Chemical Engineering, MIIT Key Laboratory of Critical Materials Technology for New Energy Conversion and Storage, Harbin Institute of Technology, Harbin 150080, China

National Key Laboratory of Science and Technology on Advanced Composites in Special Environments, and Center for Composite Materials and Structures, Harbin Institute of Technology, Harbin 150080, China

${ }^{\text {z}}$ School of Physics, University of Electronic Science and Technology of China, Chengdu 610054, China 


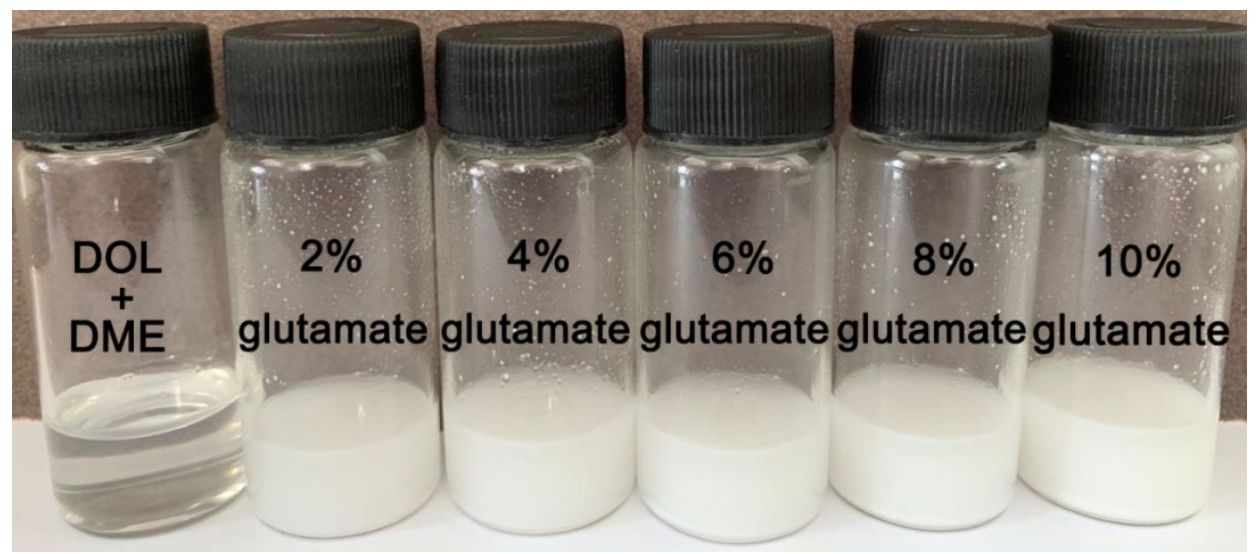

Figure S1. Photographs of DOL+DME electrolyte and glutamate additive electrolytes. 


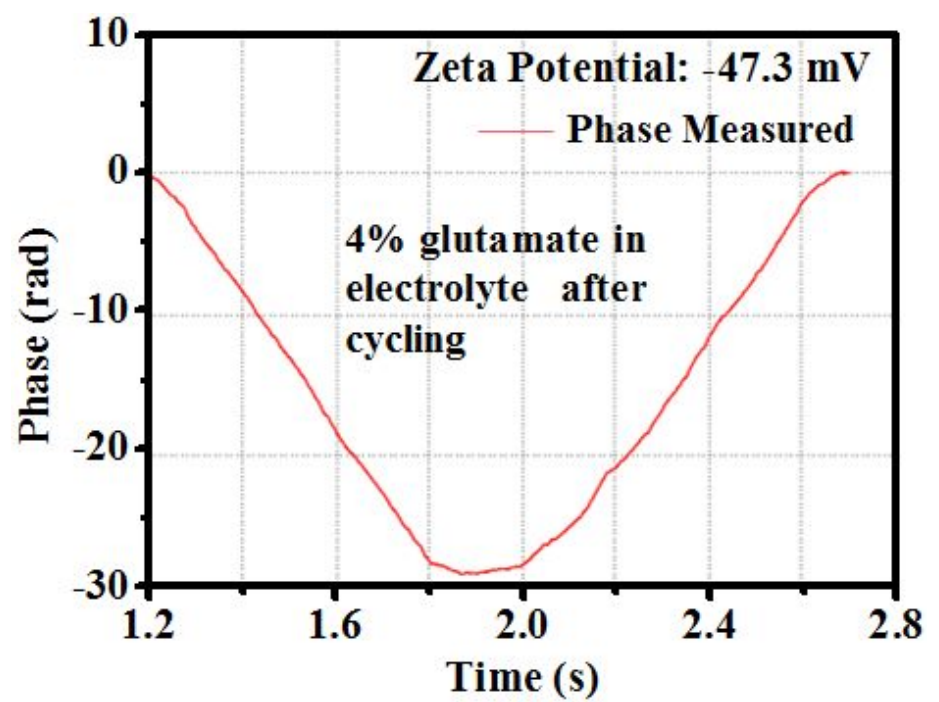

Figure S2. Zeta potential of $4 \%$ glutamate electrolyte in the Li-S battery after 100 cycles. 


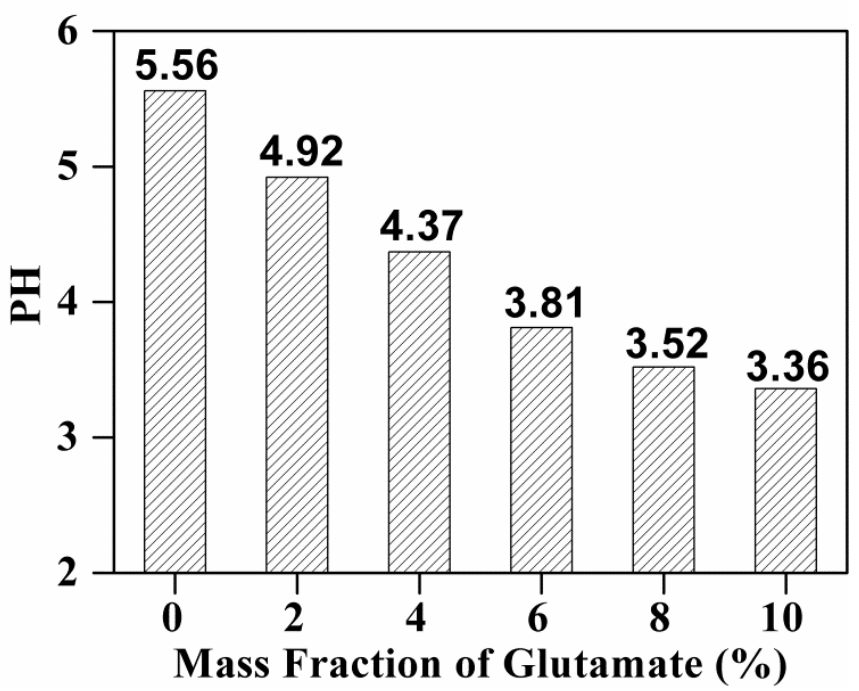

Figure S3. $\mathrm{pH}$ values of different electrolytes. 


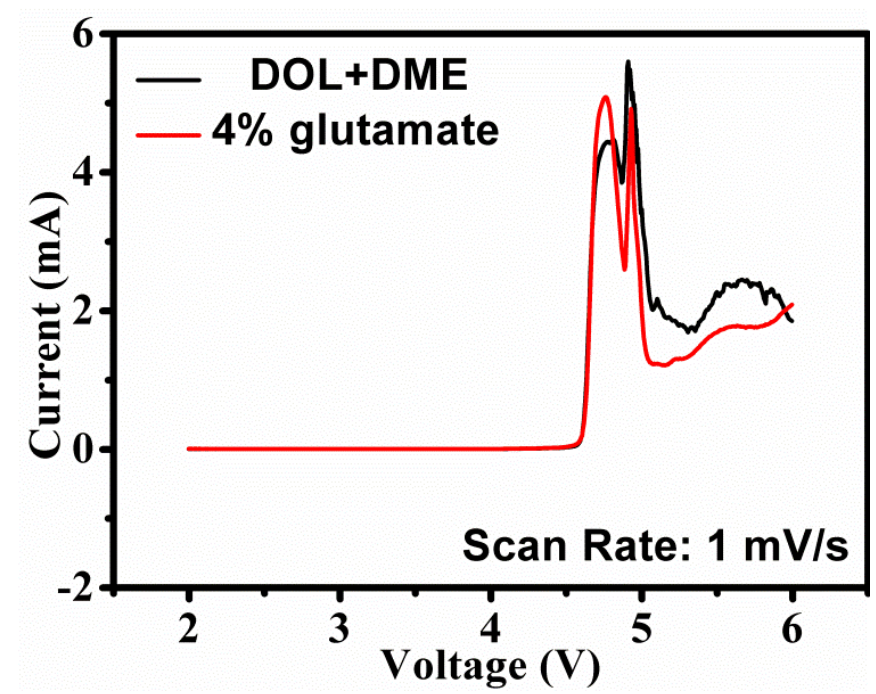

Figure S4. LSV of DOL+DME and $4 \%$ glutamate electrolytes. 

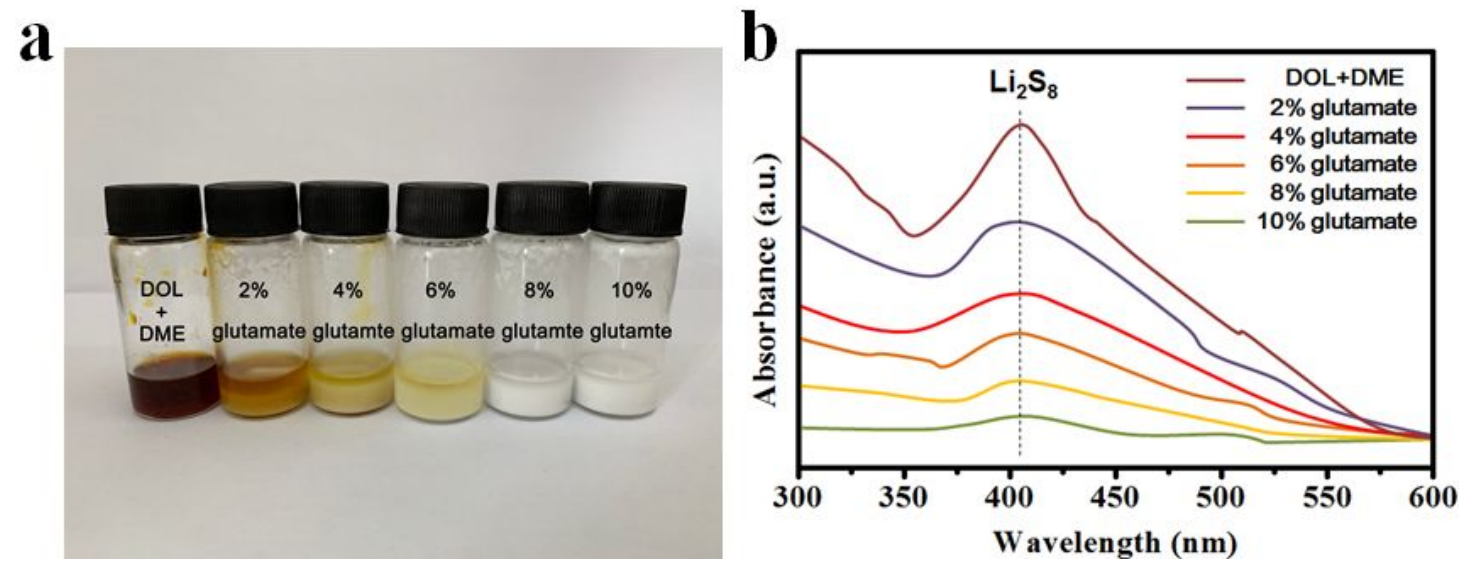

Figure S5. a, Optical images and b, UV-vis absorption spectra of saturated $\mathrm{Li}_{2} \mathrm{~S}_{8}$ in different electrolytes after $48 \mathrm{~h}$. 

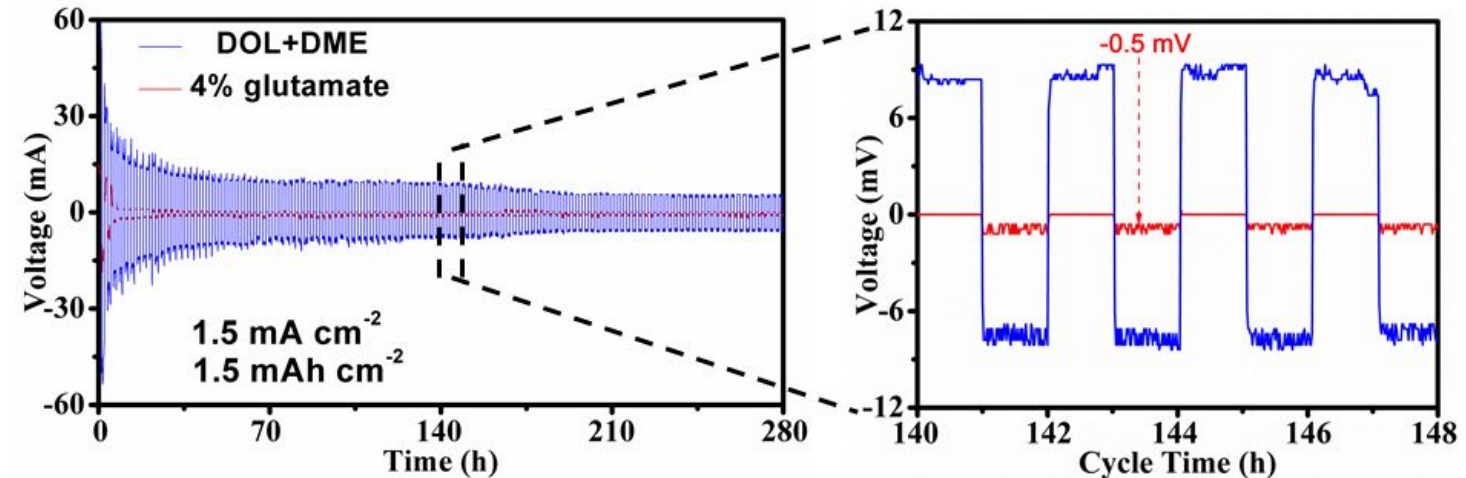

Figure S6. Cycling performance comparisons of Li| Li symmetric cells with and without glutamate additives at current densities of $1.5 \mathrm{~mA} \mathrm{~cm}^{-2}$ with a fixed capacity of 1.5 $\mathrm{mAh} \mathrm{cm}^{-2}$. 


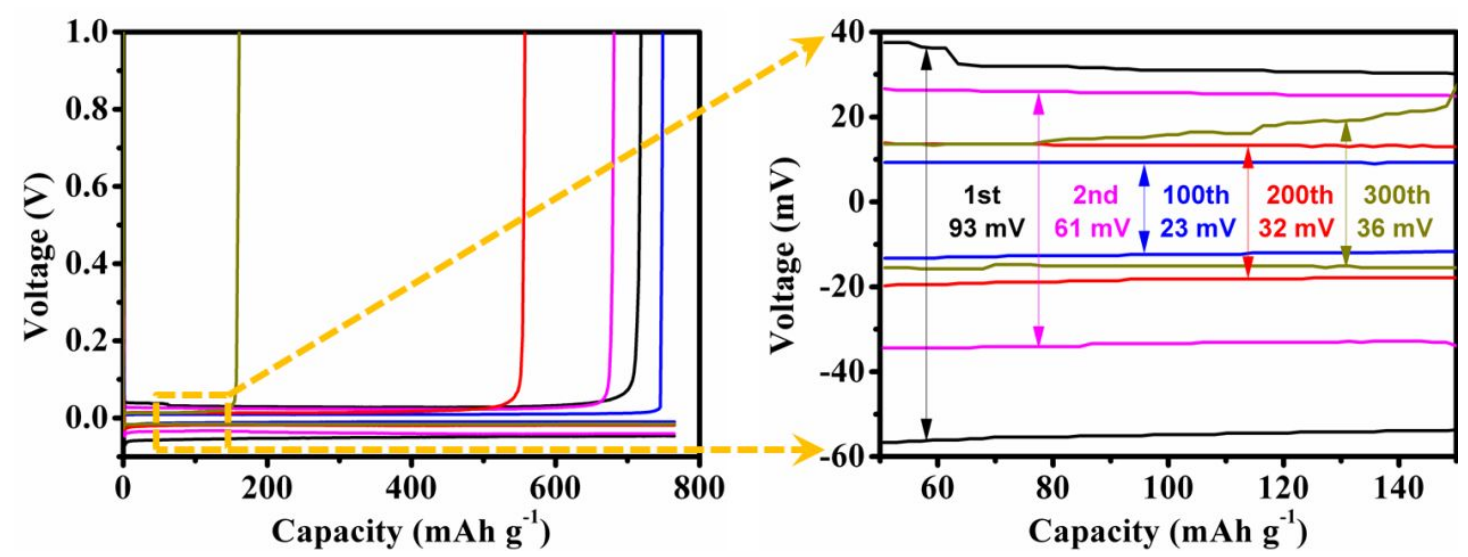

Figure S7. Voltage profiles of $\mathrm{Li}$ plating/stripping on $\mathrm{Cu}$ electrode cycled in DOL+DME electrolyte at a current density of $0.5 \mathrm{~mA} \mathrm{~cm}^{-2}$. 


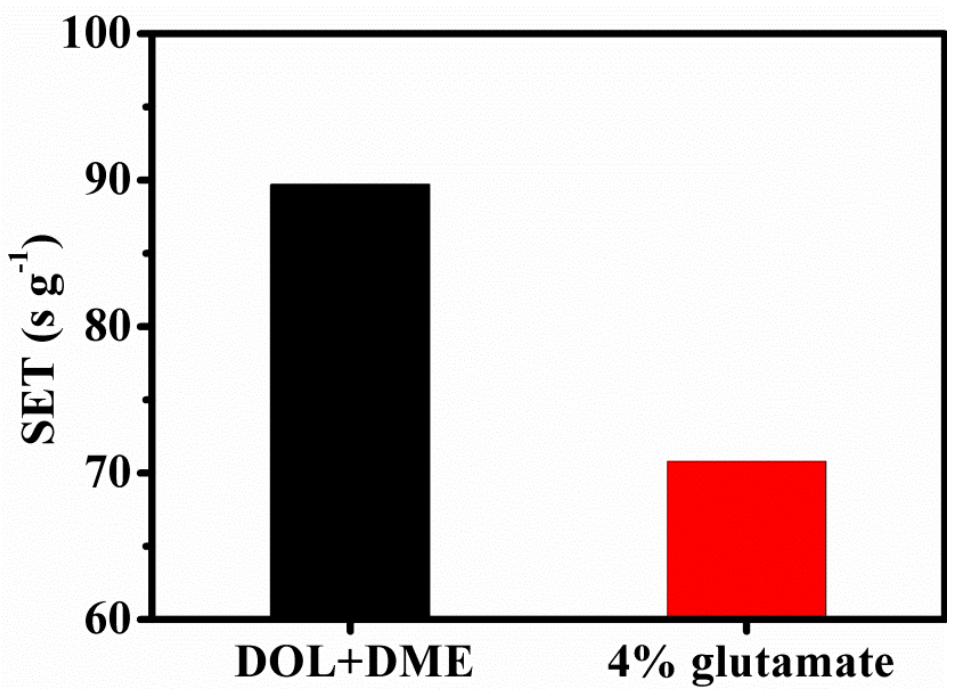

Figure S8. SET values of DOL+DME and $4 \%$ glutamate electrolytes. 


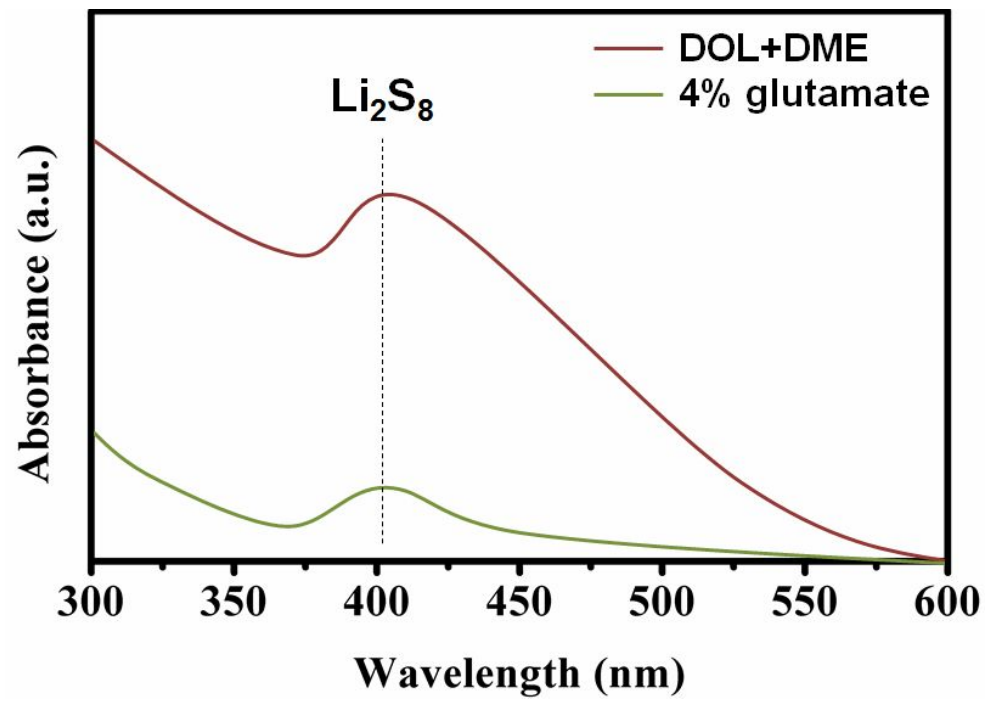

Figure S9. UV-vis spectra of $\mathrm{Li}_{2} \mathrm{~S}_{8}$ solutions with DOL+DME and $4 \%$ glutamate electrolytes. 


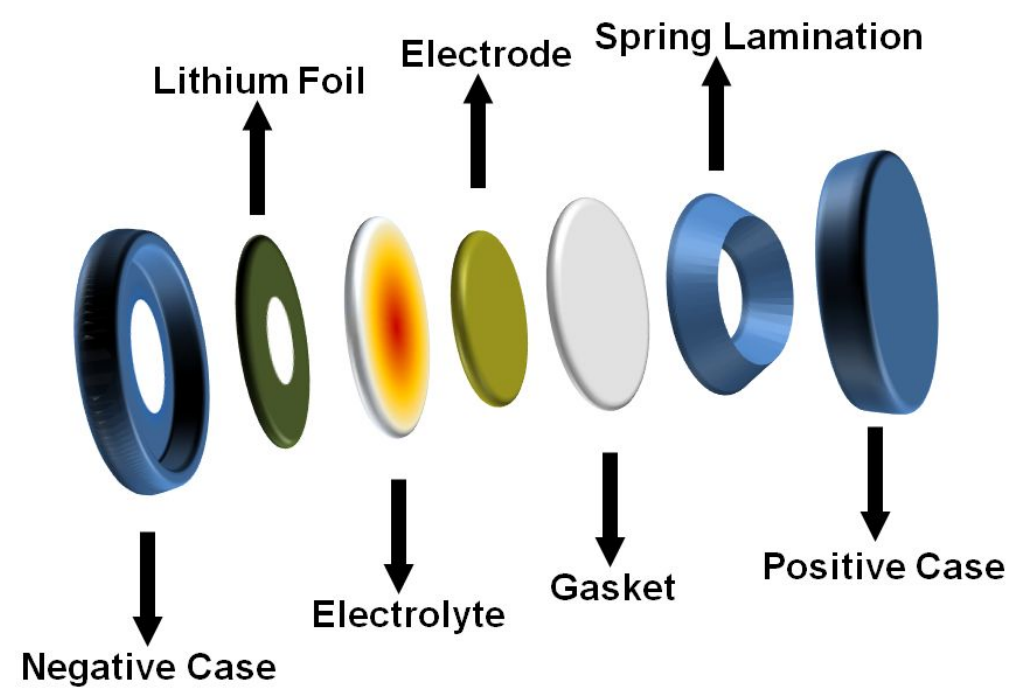

Figure S10. The cell configuration for in-situ Raman spectroscopy analyses. 


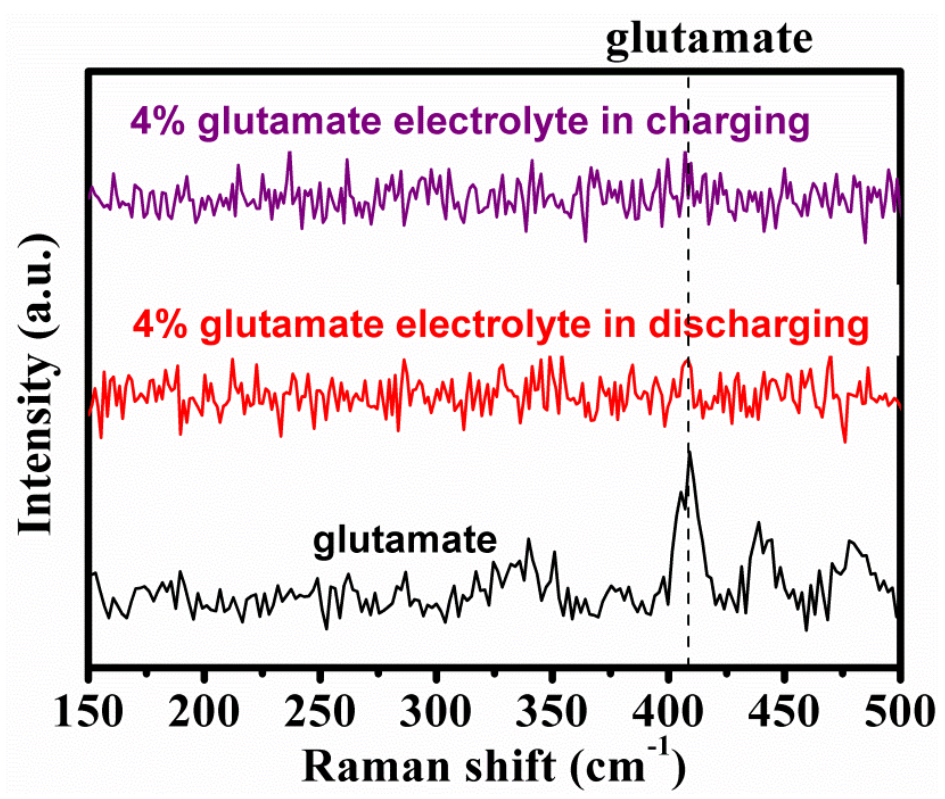

Figure S11. Raman spectra of glutamate and $4 \%$ glutamate electrolyte. 

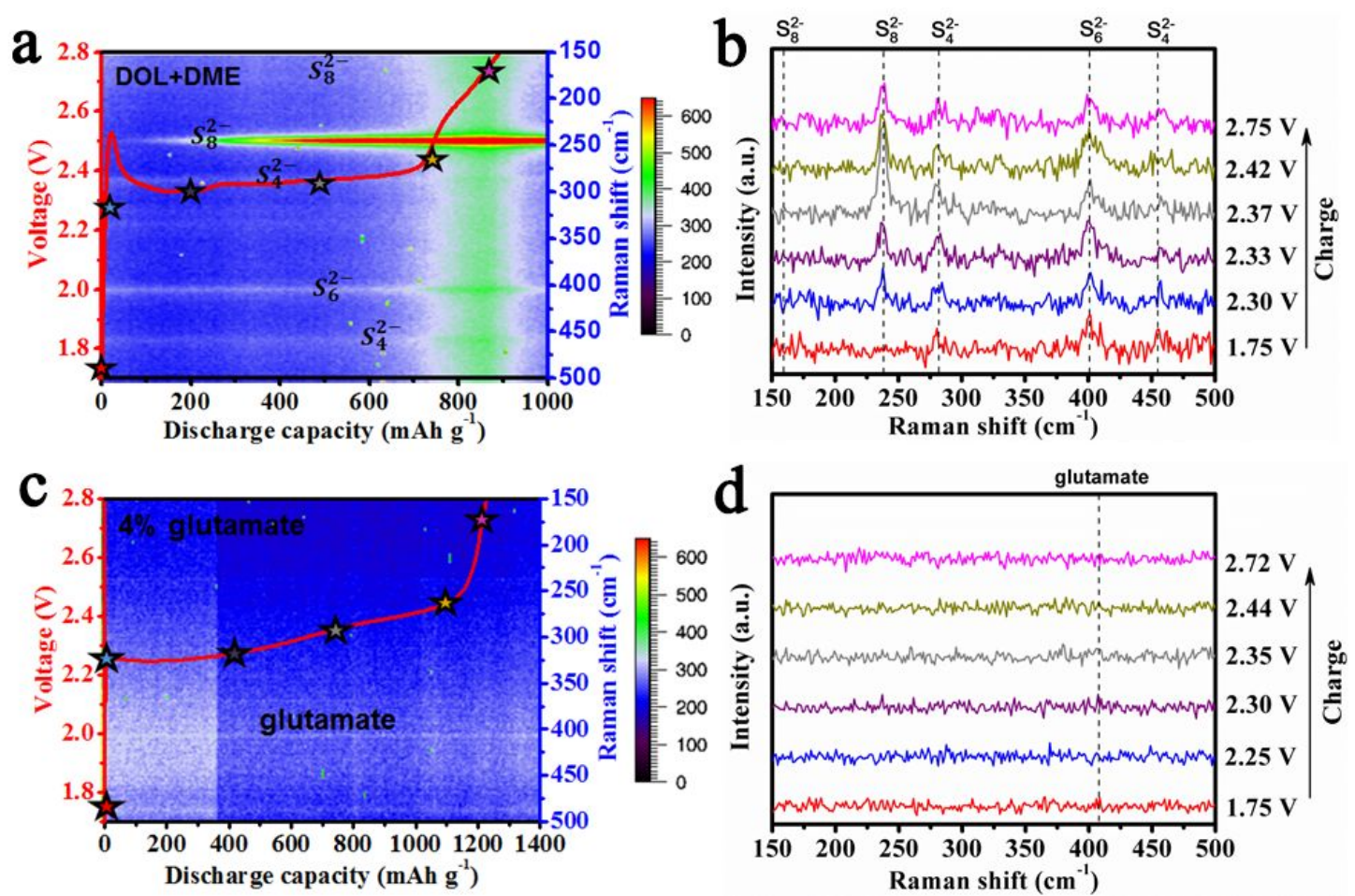

Figure S12. a, c, Time-resolved Raman spectra at $0.2 \mathrm{C}$ during charging processes of the batteries with DOL+DME and $4 \%$ glutamate electrolytes. The red curves in and c are the voltage profiles of Li-S batteries with DOL+DME and $4 \%$ glutamate electrolytes. b, d, Selected Raman spectra of Li-S batteries with DOL+DME and $4 \%$ glutamate electrolytes. 


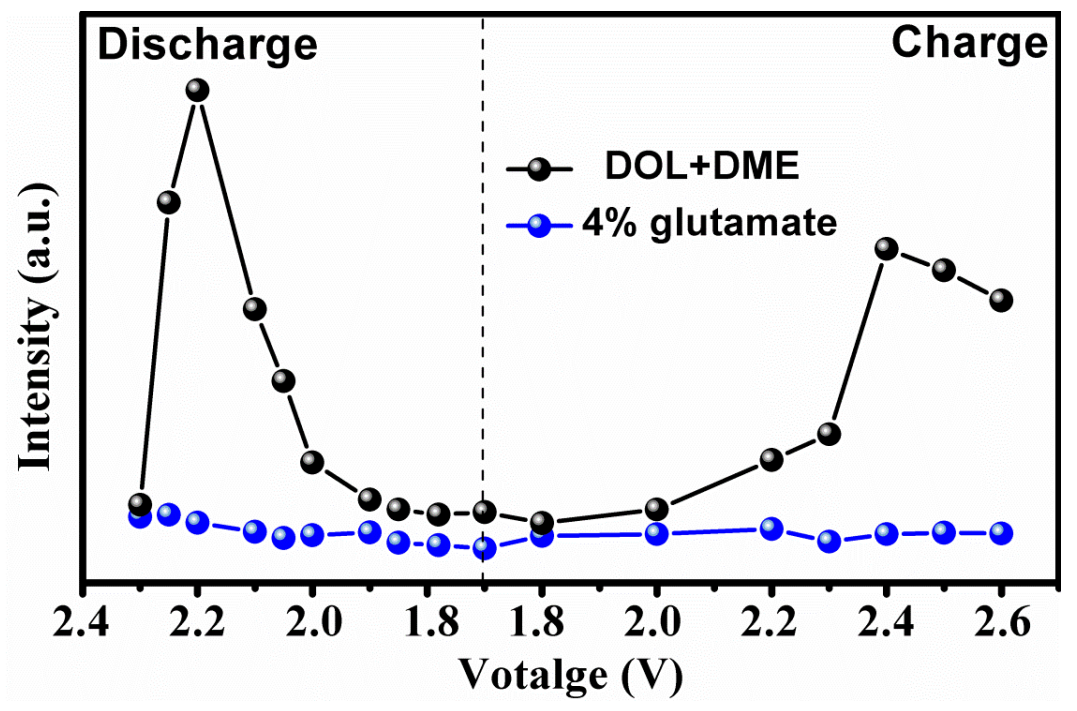

Figure S13. Comparison of $\mathrm{Li}_{2} \mathrm{~S}_{8}\left(228 \mathrm{~cm}^{-1}\right)$ concentration variations for the cells with DOL+DME and $4 \%$ glutamate electrolytes during the discharging-charging processes. 

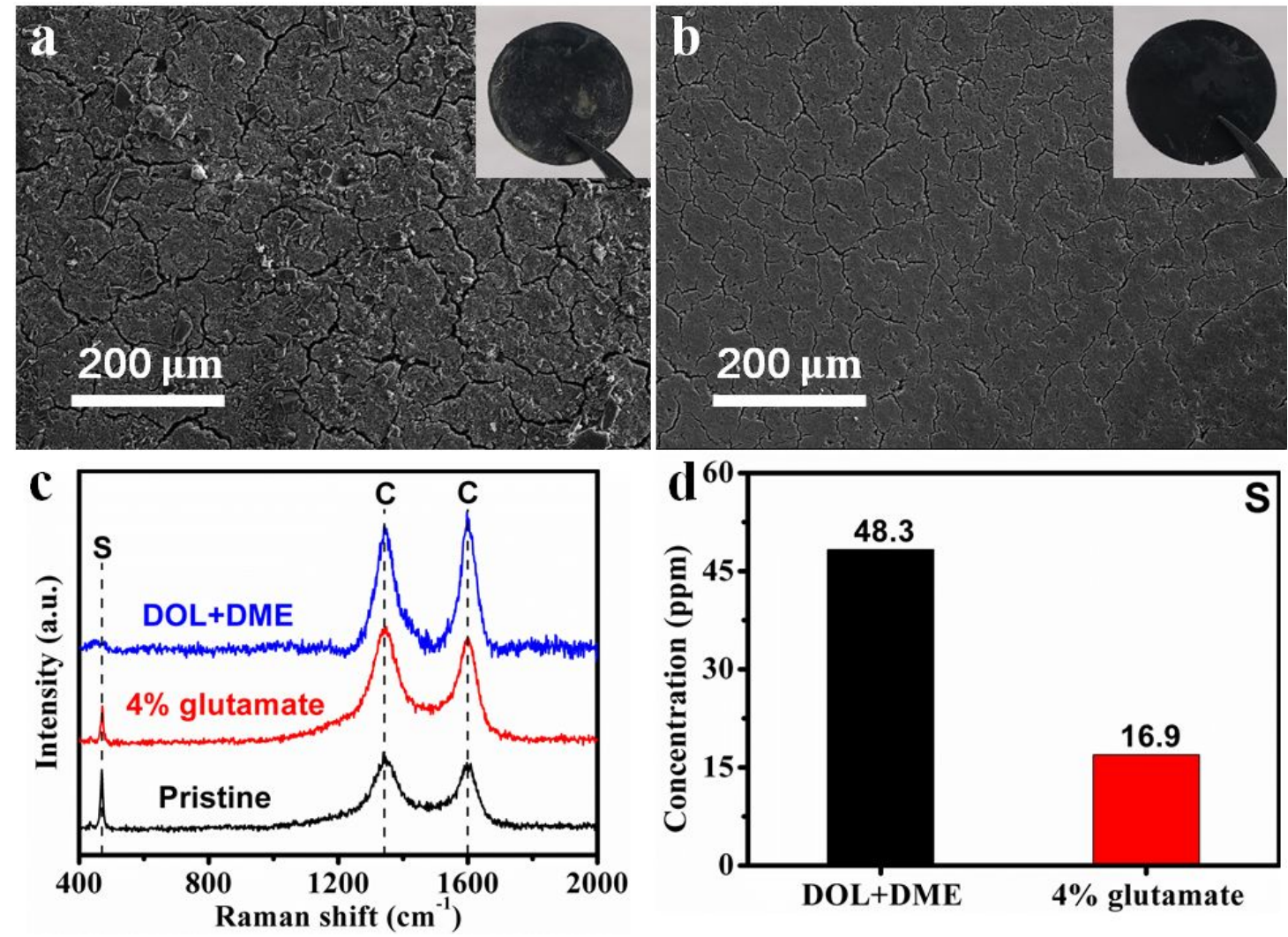

Figure S14. Optical and surface SEM images of S@C composite cathodes after 100 cycles $\mathbf{a}$, without and $\mathbf{b}$, with glutamate additive. $\mathbf{c}$, Raman spectra of pristine and postcycle S@C composite cathodes after 100 cycles without and with glutamate additive. d, Inductively coupled plasma (ICP) results of post-cycle S@C composite cathodes with DOL+DME and $4 \%$ glutamate electrolytes.

Inductively coupled plasma tests: the electrodes were disassembled from Li-S batteries after 10 cycles and soaked in the DOL+DME electrolyte for $12 \mathrm{~h}$. Then the supernatant solution was taken to analyze the ion concentration. 


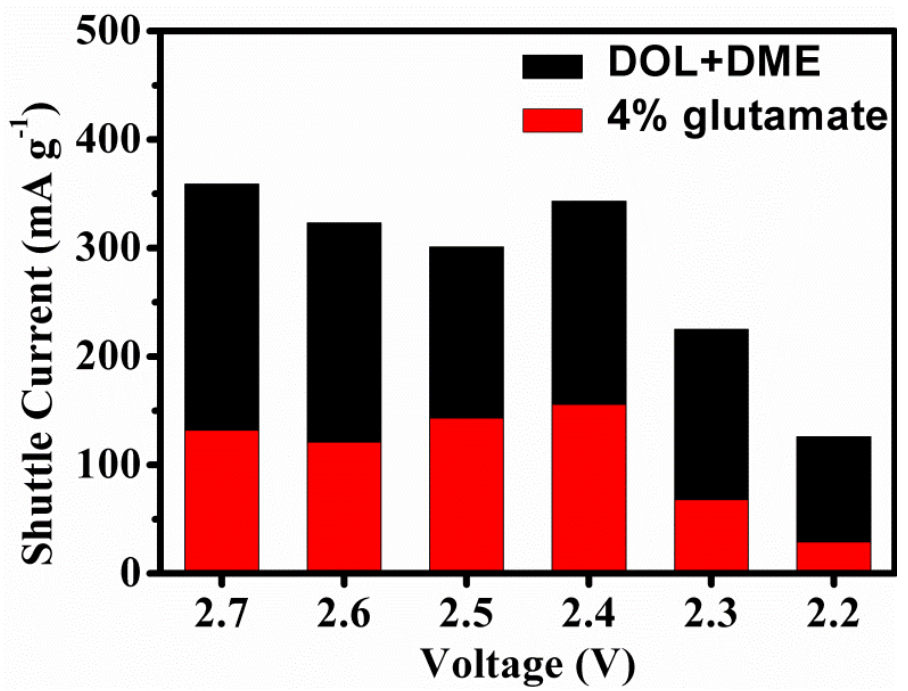

Figure S15. The comparison of shuttling currents versus applied potentiostatic charging voltages between DOL+DME and $4 \%$ glutamate electrolytes. 


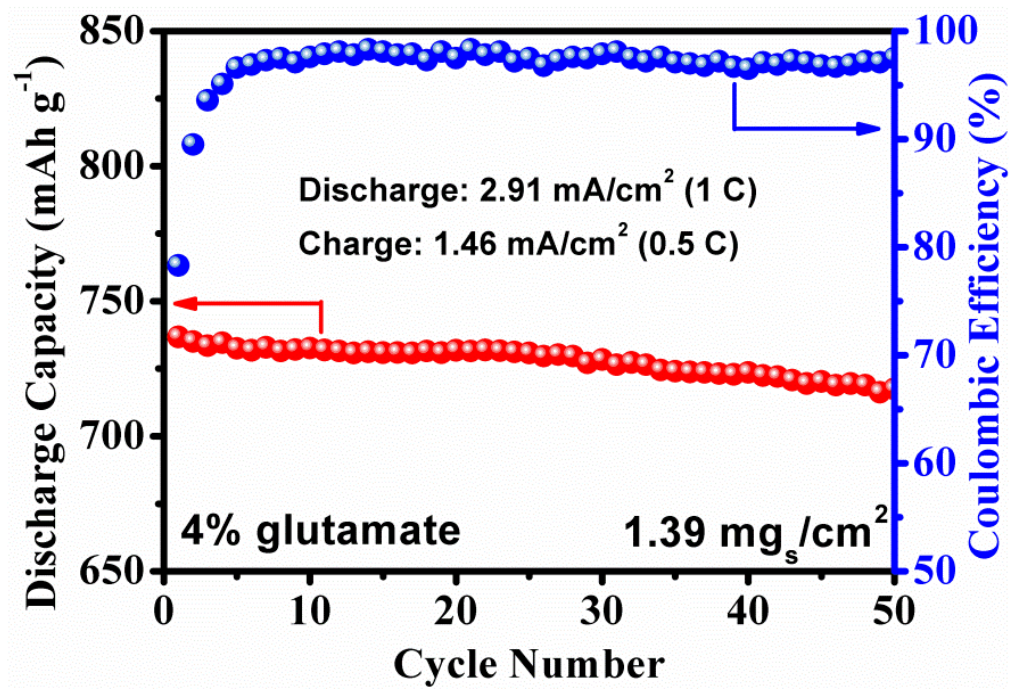

Figure S16. Cycling performances of the Li-S battery with $4 \%$ glutamate electrolyte at $0.5 \mathrm{C}$ charge and $1 \mathrm{C}$ discharge. 
a

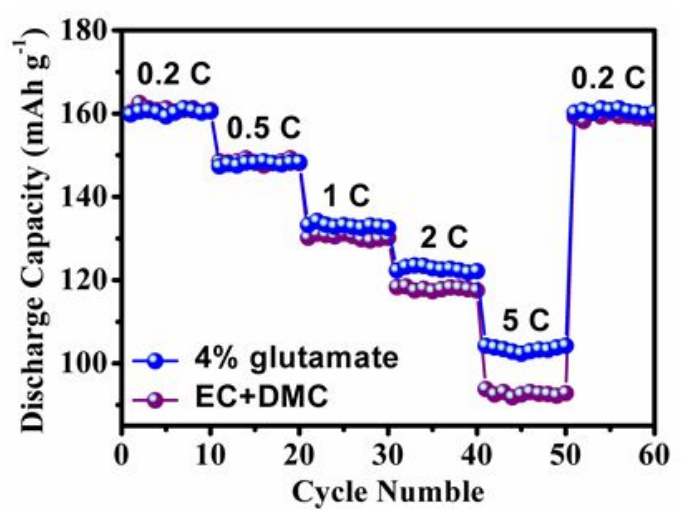

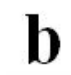

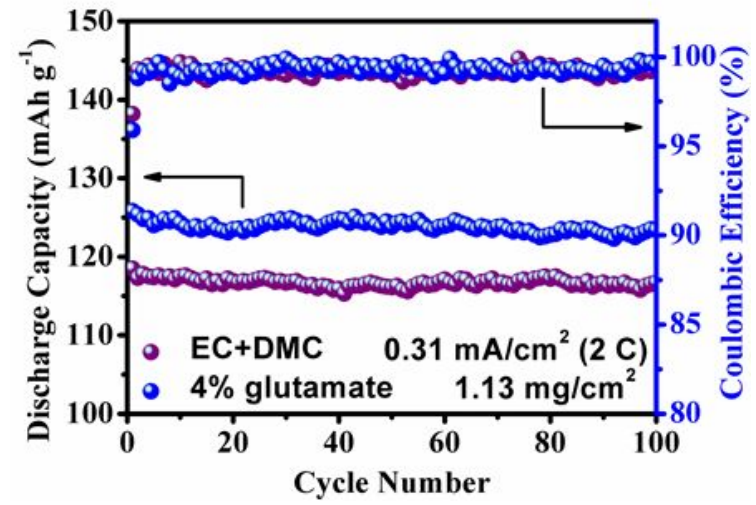

Figure S17. a, Rate performances and b, cycling performances (2 C) of the $\mathrm{LiFePO}_{4} / \mathrm{Li}$ half cells with EC+DMC and $4 \%$ glutamate electrolytes. 


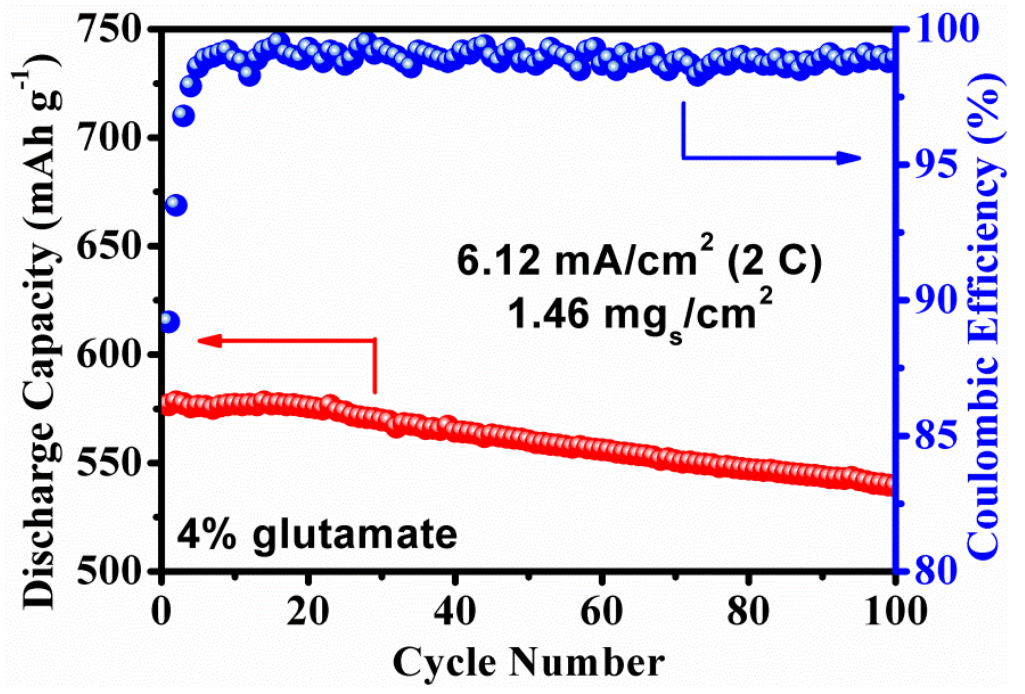

Figure S18. Cycling performances $(2 \mathrm{C})$ of the Li-S battery with $4 \%$ glutamate electrolyte and PVDF separator. 


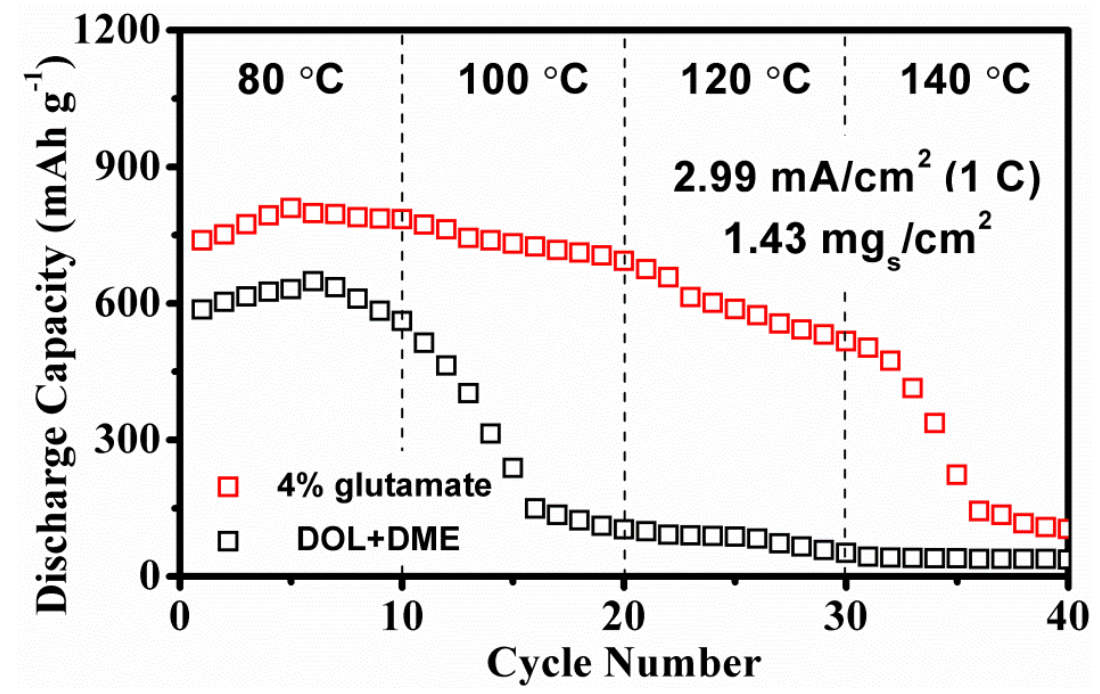

Figure S19. The cyclings of Li-S batteries with DOL + DME and $4 \%$ glutamate electrolytes at different high temperatures. 